Check for updates

Cite this: RSC Adv., 2020, 10, 556

Received 22nd October 2019

Accepted 14th December 2019

DOI: $10.1039 / \mathrm{c} 9 \mathrm{ra0} 8649 \mathrm{~h}$

rsc.li/rsc-advances

\section{Urazolium diacetate as a new, efficient and reusable Brønsted acid ionic liquid for the synthesis of novel derivatives of thiazolidine-4-ones $\dagger$}

\author{
Leila Zare Fekri, iD * Hooshang Hamidian and Masoomeh Abdollahi Chekosarani
}

\section{Introduction}

Thiazolidinone-4-ones have attracted a lot of interest because of their broad spectrum of biological activities such as anticancer, ${ }^{1,2}$ antitumor, ${ }^{3,4}$ antimicrobial, ${ }^{5}$ anti-inflammatory, ${ }^{6}$ antifungal, ${ }^{7}$ anti-malarial, ${ }^{8}$ herbicidal, ${ }^{9}$ anti-viral, ${ }^{10}$ anti-proliferative, ${ }^{11}$ and anti-Toxoplasma gondii activities. ${ }^{12}$ Furthermore, compounds containing a thiazolidine nucleus have properties such as anti-HIV ${ }^{13}$ anti-convulsant, ${ }^{14}$ cycloxygenase inhibitory, ${ }^{15}$ anti-histaminic, ${ }^{16}$ anti-platelet activating factor, ${ }^{17} \mathrm{Ca}^{2+}$ channel blocker, ${ }^{18}$ anti-oxidant, ${ }^{19,20}$ anti-tubercular, ${ }^{21}$ and analgesic ${ }^{22}$ properties.

Several methods for the synthesis of 4-thiazolidinones are widely reported in the literature. The main synthetic routes to synthesize 1,3-thiazolidin-4-ones involve three components reaction between amine, a carbonyl compound and a mercaptoacid. ${ }^{23-25}$ The other protocols for the synthesis of thiazolidine-4ones include (1) one-step cyclization reaction between ethyl 5phenylthioureido-3H-imidazole-4-carboxylate and bromoacetic acid to afford (imidazolylimino)thiazolidinones, ${ }^{26}(2)$ the reaction of aryl or alkyl isothiocyanate with a primary amine followed by treating with halo acetic acid to give 2-iminothiazolidin-4-ones, ${ }^{27}$ (3) coupling reaction between $\alpha$-chloro amide derivatives with isothiocyanate in the presence of a mild base to afford the iminothiazolidinone derivatives, ${ }^{28}$ (4) the reaction between $N$-aryl- $N$-acyl thioureas and dimethyl acetylenedicarboxylate for the preparation of thiazolidine-5-ylidenes under microwave condition, ${ }^{29}$ (5) multicomponent synthesis of thiazolidinones using ethyl 3-aminopropionate hydrochloride, aldehydes and thioglycolic acid, ${ }^{29},(6)$ the treatment of (4methyl-2-oxo-2H-chromen-7-yloxy)acetic acid hydrazide,

Department of Chemistry, Payame Noor University, PO Box 19395-3697, Tehran, Iran. E-mail:chem_zare@yahoo.com; chem_zare@pnu.ac.ir

$\dagger$ Electronic supplementary information (ESI) available. See DOI: $10.1039 / \mathrm{c} 9 \mathrm{ra} 08649 \mathrm{~h}$ aldehydes and thioglycolic acid in the presence of $\mathrm{ZnCl}_{2}$ under refluxing in dioxane ${ }^{30}$ the multicomponent synthesis of thiazolidinones under microwave reaction using various anilines, aldehydes and thioglycolic acid, ${ }^{29}$ the reaction between $\mathrm{N}$-aryl-2chloroquinolin-3-yl-azomethine and thioglycolic acid in the presence of zeolite under microwave irradiation. ${ }^{29}$

One of the twelve principles of green chemistry is avoiding of the use of auxiliary substances such as solvents and separation agents. ${ }^{31}$ The toxic and hazardous properties of many solvents suppose crucial environmental concerns such as atmospheric emissions and contamination of water effluents. It is recognized that the use of nonconventional solvents as alternatives for environmentally unfriendly traditional solvents can reduce waste solvent production and hence reduce the negative impact on environment. ${ }^{32}$ The most prevalent of these alternative solvents are water, supercritical fluids (like supercritical $\mathrm{CO}_{2}$ ), ionic liquids and solvent-free processes. ${ }^{33,34}$

Among the proposed nonconventional solvents, the use of ionic liquids in organic synthesis is interesting. Also, functional ionic liquids referred "task specific ionic liquids (TSIL)" are developing. ${ }^{35}$ The term of TSIL or functionalized ionic liquids actually indicates the ability of ionic liquids to act as catalyst and media, both. The application of acidic (Brønsted as well as Lewis) task specific ionic liquids (TSILs) as a catalytic system is growing rapidly in the field of catalysis. ${ }^{35,36}$ Combining the useful characteristics of solid acids and mineral acids, reusable TSILs have been synthesized to be applied instead of the traditional harmful mineral liquid acids, such as hydrochloric acid and sulphuric acid in the chemical reactions. However, these aspects have inadequately led many stakeholders to qualify ILs as 'green solvents'. This qualification was stated without sufficient caution about versatility of ILs. Chemical structures which could induce significant variation in risk profiles, for example, inherently combined hydrophilicity/ hydrophobicity profile of ILs and limited chemical stability of some common formulations of interest might favor diffuse or 
accidental contamination of aquatic environment through interactions of effluents or due to accidental spills. Linked to the increase of applications and the risk of environmental contamination, growing concerns have been raised on their potential environmental and health risks. Recent studies have focused on risks of ILs using various aquatic organisms and some of them have highlighted the interest to address their immunotoxicity. ${ }^{37-41}$

In view of the importance of the thiazolidinone nucleus, there is a lot of interest to accommodate the new generation of this heterocyclic moiety together with introduction a novel task specific ionic liquid "urazolium diacetate".

\section{Result and discussion}

As a part of our previous interest towards the synthesize new heterocyclic and pharmaceutical compounds ${ }^{42-49}$ and introduction new avenues and catalysts ${ }^{50-52}$ in the organic transformations, here, we report a facile, green, new and efficient task specific ionic liquid urazolium diacetate for the synthesize novel thiazolidine-4-ones through three component reaction of various aldehydes, thioglycolic acid and 4-aminoazobenzene for the first time.

Initially, TSIL urazolium diacetate was synthesized by the reaction between urazole and excess acetic acid under heating at $80{ }^{\circ} \mathrm{C}$ for $4 \mathrm{~h}$ (Scheme 1). The yellow-orange-like TSIL was washed with $3 \times 10 \mathrm{~mL}$ diethylether to departure of unreacted materials. After drying of the TSIL under vacuum, the synthesized urazoliumdiacetate IL was analyzed and characterized by FT-IR and ${ }^{1} \mathrm{H}$ NMR. In the FT-IR spectrum, the carbonyl moiety of acetate and urazolium was appeared at $1787 \mathrm{~cm}^{-1}$ as a strong and slightly broad peak because of overlap between two corresponding carbonyls and, also the unreacted $\mathrm{NH}$ group was appeared at $3270 \mathrm{~cm}^{-1}$. In ${ }^{1} \mathrm{H}$ NMR spectrum, the hydrogen atoms of methyl in acetate was shown as a singlet at $2.33 \mathrm{ppm}$ and 5 hydrogen atoms of dicationic piece of ionic liquid were appeared as a singlet at $10.65 \mathrm{ppm}$ with integral equal to 4 . One hydrogen atom in the dicationic section of IL was exchanged with broadening in the spectra base line.

The activity of the ionic liquid as a catalyst was then investigated by employing it in the multicomponent synthesis of new derivatives of thiazolidine-4-ones (Scheme 2).

To check the efficiency of urazoliumdiacetate in this reaction, 4-nitrobenzaldehyde $(1.0 \mathrm{mmol})$, thioglycolic acid (1.0 $\mathrm{mmol})$, and 4 -aminoazobenzene $(1.0 \mathrm{mmol})$ was attempted in

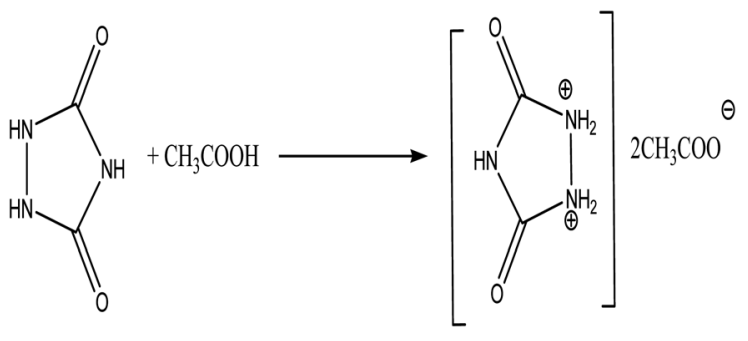

Scheme 1 Synthesis of ionic liquid urazolium diacetate. different catalysts under stirring and also in various solvents. None of the desired 2-(4-nitrophenyl)-3-(4-(phenyldiazenyl) phenyl)thiazolidin-4-one $4 a$ was obtained after stirring the reaction mixture for $24 \mathrm{~h}$ at room temperature in the absence of catalyst (Table 1 , entry 1 ). The reactions were carried out in the presence of various catalysts as $\mathrm{HCl}, \mathrm{K}-10, \mathrm{HY}$-zeolite, $\mathrm{Fe}_{3} \mathrm{O}_{4}$, [BMIM] Br and [DBU] OAc and show low yield of product $4 \mathbf{a}$ after reported reaction times (Table 1 , entries 2-7). The yield of the product $4 a$ improved remarkably to $98 \%$ in the presence of new synthesized ionic liquid urazoliumdiacetate (Table 1 , entry 8 ). The multicomponent reaction was attempted in various amounts of the catalyst and media (Table 1, entries 8-11). The results revealed that this multicomponent reaction lead to the product $\mathbf{4 a}$ in higher yield and shorter reaction time using $0.3 \mathrm{ml}$ ionic liquid per $1 \mathrm{mmol}$ substrate.

To expand the scope and generality of the application of urazoliumdiacetate in this reaction, various benzaldehydes were reacted with 4-aminoazobenzene and thioglycolic acid. The results are summarized in Table 2 . Electron-withdrawing groups on aldehydes showed increased yields in comparison to electron-releasing groups. It is because of the increasing electrophilic properties of carbon atom of carbonyl group of aldehyde bearing electron withdrawing moiety for nucleophilic addition reaction. As shown in Table 2, to control the efficiency of this method some pyrazole carbaldehydes were synthesized and undergo the multicomponent reaction with anilines and thioglycolic acid. The anilines with steric hindrance in substituents carried out the reaction in higher reaction time. Anilines with electron releasing moiety shorten the reaction time.

All of new synthesized thiazolidin-4-ones were characterized by FT-IR, ${ }^{1} \mathrm{H}$ NMR, ${ }^{13} \mathrm{C}$ NMR and elemental analysis. Because of poor solubility in some cases (4a, $\mathbf{4 e}$ and $\mathbf{4 j}$ ), unfortunately, taking ${ }^{13} \mathrm{C}$ NMR was impossible, for this reason, we were interested in taking mass spectra instead of ${ }^{13} \mathrm{C}$ NMR.

In order to demonstrate the potential application of this route, the reaction was carried out on a gram scale. As shown in Scheme 3, when $1.5 \mathrm{~g}$ of $\mathbf{1 a}$ was used under the standard conditions, the product 4 a was obtained in $98 \%$ yield with the reaction time prolonged to $25 \mathrm{~min}$, indicating that this route could be scaled up to a preparing scale.

As proposed in Scheme 4, initially, the carbonyl moiety of aldehyde and amino group was activated by urazoliumdiacetate by dipolarization to facilitate the nucleophilic attack of amine to carbonyl group. Then, the imine moiety was produced via elimination of $\mathrm{H}_{2} \mathrm{O}$. Nucleophilic attack of activated sulphur to imine followed by intramolecular nucleophilic attack of secondary amine to carboxylic acid moiety lead to product 4 . The mechanistic pathway was supported by literature..$^{30,53}$

After reaction, the ionic liquid is easily separated from the reaction medium by washing with distilled water (IL is soluble in water). The washed ionic liquid is distilled under vacuum to recover solvent for reuse in subsequent reactions. After seven successive runs, recycled ionic liquid showed suitable efficiency with regard to reaction time and yield (Fig. 1). 


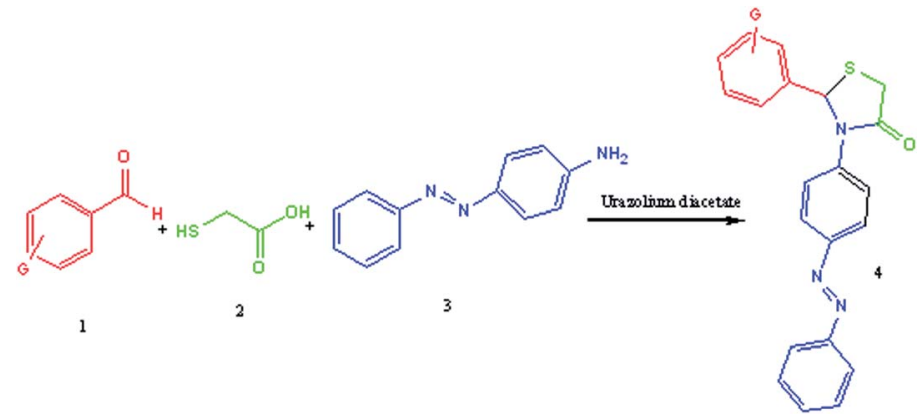

Scheme 2 Urazolium diacetate catalyzed synthesis of azo dispersive dyes-thiazolidine.

\section{Conclusion}

In conclusion, we synthesize and apply urazolium diacetate as a novel Brønsted dicationic acid for the preparation of new azo dispersive dyes and pyrazolyl compounds with thiazolidine-4one moiety. The notable benefits of this synthesis are: catalyst is inexpensive, non-toxic, easy handling, green and reusable. Simple work-up procedure, short reaction time and high yields of product, together with avoiding toxic and hazardous solvent are the other advantages. To the best of our knowledge, the ionic liquid urazolium diacetate and all of synthesized azo dispersive dyes and pyrazolyl compounds with thiazolidine-4one moiety are completely new and this is the first report for the synthesis of thiazolidine-4-ones using ionic liquid urazolium diacetate.

\section{Experimental section}

\section{Materials and methods}

Chemicals were purchased from Merck and Fluka. All solvents used were dried and distilled according to standard procedures. Melting points were measured on an Electrothermal 9100 apparatus. IR spectra were determined on a Shimadzu FT-IR 8600 spectrophotometer. ${ }^{1} \mathrm{H}$ and ${ }^{13} \mathrm{C}$ NMR spectra were determined on a Bruker DRX Avance instrument at 500 or 300 and 125 or $75 \mathrm{MHz}$. HR-MS data were taken by Quadropole 5975C made by Agilent technologies.
The procedure for the synthesis of ionic liquid urazoliumdiacetate

A mixture of $1 \mathrm{mmol}$ of urazole and excess amount of acetic acid were heated at $80{ }^{\circ} \mathrm{C}$ for 4 hours to obtain a yellow-orange like oil. The corresponding oil was washed and decanted with diethylether $(3 \times 10 \mathrm{~mL})$ to departure of the unreacted materials. The residue was dried under vacuum to reach pure ionic liquid. The ionic liquid was synthesized for the first time and characterized by FT-IR and ${ }^{1} \mathrm{H}$ NMR. FT-IR $\left(\mathrm{KBr}, \mathrm{cm}^{-1}\right): 3270$, 3020, 1787, 1467, and 1235. ${ }^{1} \mathrm{H}$ NMR (DMSO-d $\left.6,500 \mathrm{MHz}\right): \delta_{\mathrm{H}}$; $2.33(\mathrm{~s}, 6 \mathrm{H}), 10.65(\mathrm{~s}, 4 \mathrm{H}) \mathrm{ppm}$.

\section{General procedure for the synthesis of 1,3-thiazolidine-4-ones}

A mixture of arylaldehyde (1 mmol), 4-aminoazobenzene (1 $\mathrm{mmol})$, thioglycolic acid and urazolium diacetate $(0.3 \mathrm{mmol})$ was heated at $80{ }^{\circ} \mathrm{C}$ for the required reaction times as indicated in Table 2. After completion of the reaction, as indicated by TLC, the reaction mixture was dissolved in $20 \mathrm{~mL}$ of $\mathrm{H}_{2} \mathrm{O}$. The product was separated by filtration and recrystallized from EtOH and dried to afford crystalline compounds of $\mathbf{4 a - m}$. Then, the ionic liquid was recovered for subsequent use. All of synthesized compounds were characterized by their physical constant, IR, NMR, mass spectroscopy and elemental analysis.

\section{Analytical data for selected compounds}

2-(4-Nitrophenyl)-3-(4-(phenyldiazenyl)phenyl)thiazolidin-4one (4a). Mp 242-244 ${ }^{\circ} \mathrm{C}$, FT-IR $\left(\mathrm{KBr}, \mathrm{cm}^{-1}\right): 1630(\mathrm{C}=\mathrm{O}$

Table 1 The optimization of catalyst and reaction condition for the synthesis of $4 a$

\begin{tabular}{|c|c|c|c|c|c|}
\hline Entry & Catalyst & Solvent/condition & Catalyst loading & Reaction time & $\begin{array}{l}\text { Reaction yield } \\
(\%)\end{array}$ \\
\hline 1 & - & -/reflux & - & $24 \mathrm{~h}$ & - \\
\hline 2 & $\mathrm{HCl}$ & EtOH/reflux & 10 drops & $14 \mathrm{~h}$ & 36 \\
\hline 3 & $\mathrm{~K}-10$ & EtOH/reflux & $0.1 \mathrm{~g}$ & $8 \mathrm{~h}$ & 42 \\
\hline 4 & HY-zeolite & EtOH/reflux & $0.1 \mathrm{~g}$ & $8 \mathrm{~h}$ & 45 \\
\hline 5 & $\mathrm{Fe}_{3} \mathrm{O}_{4}$ & EtOH & $0.05 \mathrm{~g}$ & $6 \mathrm{~h}$ & 52 \\
\hline 6 & {$[\mathrm{BMIM}] \mathrm{Br}$} & {$[\mathrm{BMIM}] \mathrm{Br} / \mathrm{heat}, 80^{\circ} \mathrm{C}$} & $0.5 \mathrm{~mL}$ & $2 \mathrm{~h}$ & 65 \\
\hline 7 & {$[\mathrm{DBU}] \mathrm{OAc}$} & {$[\mathrm{DBu}] \mathrm{OAc} / \mathrm{heat}, 80^{\circ} \mathrm{C}$} & $0.5 \mathrm{~mL}$ & $2.5 \mathrm{~h}$ & 61 \\
\hline 8 & Urazolium diAc & [Urazolium]diAc/heat, $80^{\circ} \mathrm{C}$ & $0.5 \mathrm{~mL}$ & 20 & 98 \\
\hline 9 & Urazolium diAc & [Urazolium]diAc/heat, $80{ }^{\circ} \mathrm{C}$ & $0.3 \mathrm{~mL}$ & 20 & 98 \\
\hline 10 & Urazolium diAc & [Urazolium] diAc/heat, $80^{\circ} \mathrm{C}$ & $0.2 \mathrm{~mL}$ & 35 & 94 \\
\hline
\end{tabular}


Table 2 The scope of multicomponent synthesis of 4a using urazoliumdiacetate

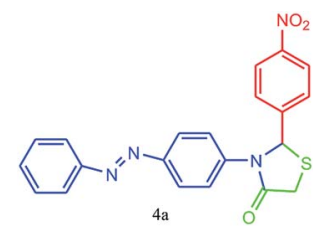<smiles>O=C1CSC(c2ccc(O)cc2)N1c1ccc(N=Nc2ccccc2)cc1</smiles><smiles>O=C1CSC(c2ccc(Br)cc2)N1c1ccc(N=Nc2ccccc2)cc1</smiles><smiles>O=C1CCC(c2ccc(Cl)cc2)N1c1ccc(N=Nc2ccccc2)cc1</smiles>

6<smiles>CN(C)c1ccc(C2SCC(=O)N2c2ccc(N=Nc3ccccc3)cc2)cc1</smiles><smiles>O=C1CSC(c2cccc([N+](=O)[O-])c2)N1c1ccc(N=Nc2ccccc2)cc1</smiles>

9<smiles>O=C1CSC(c2ccccc2Cl)N1c1ccc(N=Nc2ccccc2)cc1</smiles> 
Table 2 (Contd.)

Entry Product

Time (min)

Yield $^{a, b}(\%)$

$\operatorname{Mp}\left({ }^{\circ} \mathrm{C}\right)$

10<smiles>O=C1CSC(c2cccc(Br)c2)N1c1ccc(N=Nc2ccccc2)cc1</smiles><smiles>O=C1CSC(c2ccccc2[N+](=O)[O-])N1c1ccc(/N=N/c2ccccc2)cc1</smiles>

12<smiles>O=C1CSC(c2ccc(Cl)cc2Cl)N1c1ccc(/N=N/c2ccccc2)cc1</smiles><smiles>COc1cc(C2SCC(=O)N2c2ccc(/N=N/c3ccccc3)cc2)ccc1O</smiles><smiles>COc1ccc(N2C(O)CSC2C2CN(c3ccccc3)N=C2c2ccc(Cl)cc2)cc1</smiles>
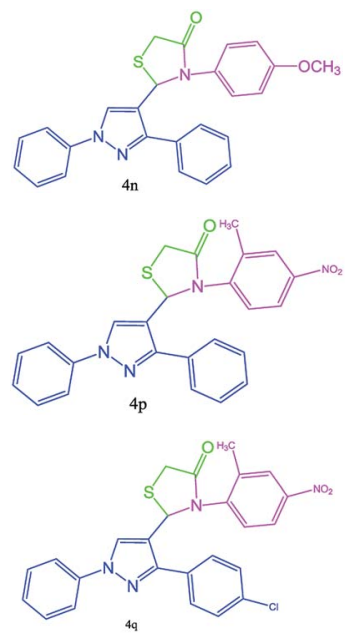
compounds are unknown and were characterized completely by various analysis.

stretching), 1590 (aromatic $\mathrm{C}=\mathrm{C}$ stretching or $\mathrm{N}=\mathrm{N}$ stretching), $1387\left(\mathrm{NO}_{2}\right.$ symmetric stretching, $\mathrm{C}-\mathrm{N}$ or $\mathrm{C}-\mathrm{S}$ stretching) $\mathrm{cm}^{-1} .{ }^{1} \mathrm{H}$ NMR (DMSO- $\left.\mathrm{d}_{6}, 500 \mathrm{MHz}\right): \delta_{\mathrm{H}} ; 4.42(\mathrm{~s}, 2 \mathrm{H})$, $6.09(\mathrm{~s}, 1 \mathrm{H}), 6.67(\mathrm{~d}, J=8.8 \mathrm{~Hz}, 2 \mathrm{H}), 7.41(\mathrm{t}, J=7.3 \mathrm{~Hz}, 1 \mathrm{H}), 7.50$ (t, $J=8.0 \mathrm{~Hz}, 2 \mathrm{H}), 7.56-7.65(\mathrm{~m}, 2 \mathrm{H}), 7.65(\mathrm{~d}, J=8.8 \mathrm{~Hz}, 2 \mathrm{H})$, $7.73(\mathrm{~d}, J=8.5 \mathrm{~Hz}, 2 \mathrm{H}), 7.87-7.92(\mathrm{~m}, 2 \mathrm{H}) \mathrm{ppm}$. HR-MS $(\mathrm{m} / \mathrm{z}$ 404). Anal. calcd for $\mathrm{C}_{21} \mathrm{H}_{16} \mathrm{~N}_{4} \mathrm{O}_{3} \mathrm{~S}$ : C, 62.36; $\mathrm{H}, 3.99 ; \mathrm{N}, 13.85$. Found: C, 62.39; H, 3.98; N, 13.89 . 


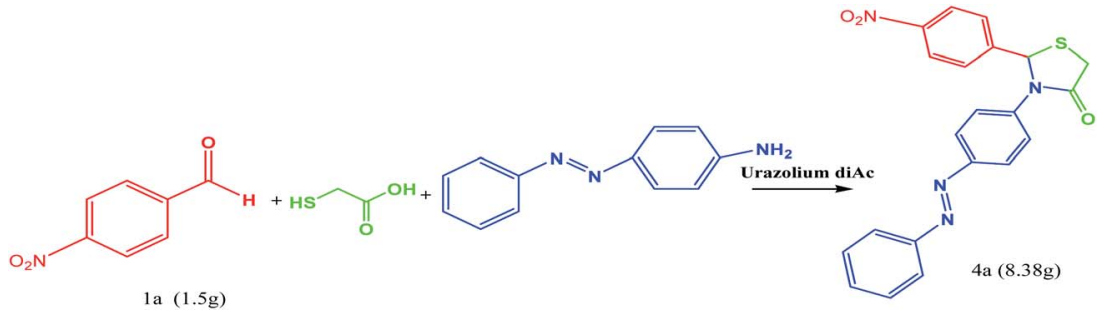

Scheme 3 The gram scale analysis for the synthesis of product $4 a$.

2-(4-Hydroxyphenyl)-3-(4-(phenyldiazenyl)phenyl)

thiazolidin-4-one (4b). Mp 221-223 ${ }^{\circ} \mathrm{C}$, FT-IR $\left(\mathrm{KBr}, \mathrm{cm}^{-1}\right): 1649$ ( $\mathrm{C}=\mathrm{O}$ stretching), 1594 (aromatic $\mathrm{C}=\mathrm{C}$ or $\mathrm{N}=\mathrm{N}$ stretching), 1450 (aromatic $\mathrm{C}=\mathrm{C}$ stretching), 1400, $1370(\mathrm{C}-\mathrm{N}, \mathrm{C}-\mathrm{S}$ or $\mathrm{C}-\mathrm{O}$ stretching) $\mathrm{cm}^{-1} \cdot{ }^{1} \mathrm{H}$ NMR (DMSO-d $\left.{ }_{6}, 500 \mathrm{MHz}\right): \delta_{\mathrm{H}} ; 4.31(\mathrm{~s}, 2 \mathrm{H})$, $6.10(\mathrm{t}, J=8.8 \mathrm{~Hz}, 3 \mathrm{H}), 6.67(\mathrm{~d}, J=8.8 \mathrm{~Hz}, 2 \mathrm{H}), 7.28(\mathrm{~d}, J=$ $8.5 \mathrm{~Hz}, 2 \mathrm{H}), 7.41(\mathrm{t}, J=6.1 \mathrm{~Hz}, 1 \mathrm{H}), 7.50(\mathrm{t}, J=7.3 \mathrm{~Hz}, 2 \mathrm{H}), 7.66$ $(\mathrm{d}, J=8.8 \mathrm{~Hz}, 2 \mathrm{H}), 7.73(\mathrm{~d}, J=8.4 \mathrm{~Hz}, 2 \mathrm{H}), 9.13(\mathrm{~s}, 1 \mathrm{H}, \mathrm{OH}) \mathrm{ppm}$. ${ }^{13} \mathrm{C}$ NMR (DMSO-d $6125 \mathrm{MHz}$ ): $\delta_{\mathrm{C}} ; 62.5$ (two peaks), 113.49, 118.78, 121.09, 121.78, 125.25, 127.22, 129.12, 129.28, 129.48, 132.56, 152.52, 152.96, 187.66 ppm. HR-MS $(m / z ~ 375)$. Anal. calcd for $\mathrm{C}_{21} \mathrm{H}_{17} \mathrm{~N}_{3} \mathrm{O}_{2} \mathrm{~S}: \mathrm{C}, 67.18 ; \mathrm{H}, 4.56 ; \mathrm{N}, 11.19$. Found: $\mathrm{C}$, 67.21; H, 4.56; N, 11.17.

2-(4-Bromophenyl)-3-(4-(phenyldiazenyl)phenyl)thiazolidin4-one (4c). Mp 251-253 ${ }^{\circ} \mathrm{C}$, FT-IR $\left(\mathrm{KBr}, \mathrm{cm}^{-1}\right): 1630(\mathrm{C}=\mathrm{O}$ stretching), 1598 (aromatic $\mathrm{C}=\mathrm{C}$ stretching or $\mathrm{N}=\mathrm{N}$ stretching), $1391(\mathrm{C}=\mathrm{N} \quad$ or $\quad \mathrm{C}-\mathrm{S} \quad$ stretching $), 1070 \quad(\mathrm{C}-\mathrm{Br}$ stretching) $\mathrm{cm}^{-1} .{ }^{1} \mathrm{H}$ NMR (DMSO-d $\left.{ }_{6}, 500 \mathrm{MHz}\right): \delta_{\mathrm{H}} ; 4.45(\mathrm{~s}, 2 \mathrm{H})$, $6.09(\mathrm{~s}, 1 \mathrm{H}), 6.67(\mathrm{~d}, J=8.8 \mathrm{~Hz}, 1 \mathrm{H}), 7.27(\mathrm{~d}, J=8.5 \mathrm{~Hz}, 1 \mathrm{H}), 7.41$ $(\mathrm{t}, J=7.3 \mathrm{~Hz}, 1 \mathrm{H}), 7.50(\mathrm{t}, J=8.3 \mathrm{~Hz}, 4 \mathrm{H}), 7.54-7.60(\mathrm{~m}, 1 \mathrm{H}), 7.65$ $(\mathrm{d}, J=8.7 \mathrm{~Hz}, 2 \mathrm{H}), 7.73(\mathrm{~d}, J=9.2 \mathrm{~Hz}, 2 \mathrm{H}), 7.87(\mathrm{~d}, J=7.4 \mathrm{~Hz}$, 1H) ppm. ${ }^{13} \mathrm{C}$ NMR (DMSO-d 6 , $75 \mathrm{MHz}$ ): $\delta_{\mathrm{C}} ; 62.58$ (two peaks), 122.93, 122.97, 123.50, 124.35, 125.63, 129.05, 129.94, 131.22, 131.74, 132.47, 149.30, 152.49, 173.64 ppm. HR-MS ( $m / z ~ 437)$. Anal. calcd for $\mathrm{C}_{21} \mathrm{H}_{16} \mathrm{BrN}_{3} \mathrm{OS}$ : C, 57.54; H, 3.68; N, 9.59. Found: C, 57.55; H, 3.71; N, 9.57.

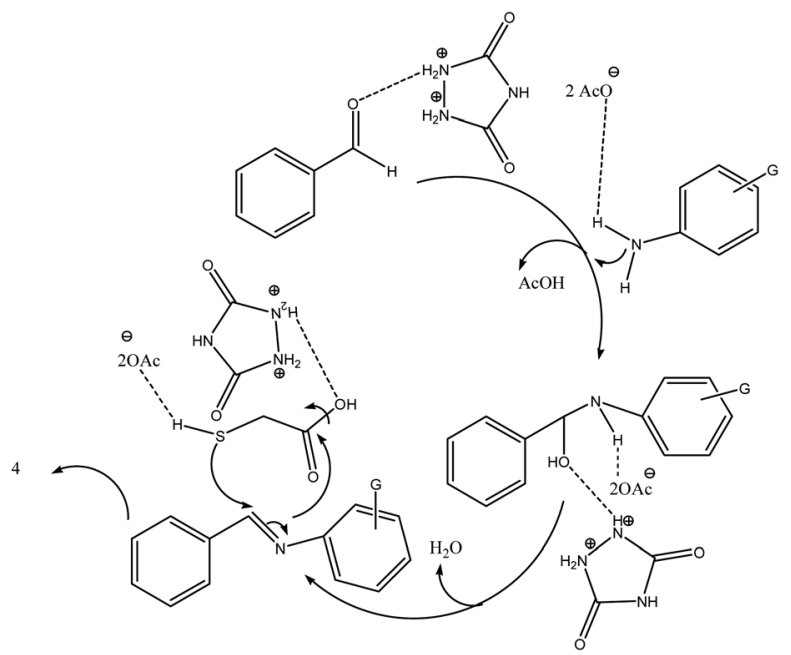

Scheme 4 Proposed mechanism of thiazolidine-4-ones using urazoliumdiacetate.
2-(4-Chlorophenyl)-3-(4-(phenyldiazenyl)phenyl)thiazolidin4-one (4d). Mp 242-244 ${ }^{\circ} \mathrm{C}$, FT-IR ( $\left.\mathrm{KBr}, \mathrm{cm}^{-1}\right): 1645(\mathrm{C}=\mathrm{O}$ stretching), 1600 (aromatic $\mathrm{C}=\mathrm{C}, \mathrm{N}=\mathrm{N}$ streching), 1421 (aromatic $\mathrm{C}=\mathrm{C}$ stretching), 1340 (C-N or C-S stretching), 1146 (C-Cl stretching) $\mathrm{cm}^{-1} \cdot{ }^{1} \mathrm{H}$ NMR (DMSO-d $\left.{ }_{6}, 500 \mathrm{MHz}\right): \delta_{\mathrm{H}} ; 4.47$ $(\mathrm{s}, 2 \mathrm{H}), 6.09$ (s, 1H), 6.67 (d, $J=8.9 \mathrm{~Hz}, 3 \mathrm{H}), 7.42(\mathrm{~d}, J=7.0 \mathrm{~Hz}$, $1 \mathrm{H}), 7.50(\mathrm{t}, J=7.9 \mathrm{~Hz}, 3 \mathrm{H}), 7.66(\mathrm{~d}, J=8.8 \mathrm{~Hz}, 3 \mathrm{H}), 7.73(\mathrm{~d}, J=$ $7.3 \mathrm{~Hz}, 3 \mathrm{H}$ ) ppm. ${ }^{13} \mathrm{C}$ NMR (DMSO-d 6 , $75 \mathrm{MHz}$ ): $\delta_{\mathrm{C}} ; 59.51,60.23$, 113.89, 116.08, 122.17, 123.67, 125.63, 128.42, 129.89, 141.23, 142.03, 143.27, 149.63, 153.32, 170.31 ppm. HR-MS ( $m / z 393)$. Anal. calcd for $\mathrm{C}_{21} \mathrm{H}_{16} \mathrm{ClN}_{3} \mathrm{OS}$ : C, 64.03; H, 4.09; N, 10.67. Found: C, 64.06; H, 4.07; N, 10.68 .

2-(4-(Dimethylamino)phenyl)-3-(4-(phenyldiazenyl)phenyl) thiazolidin-4-one (4e). Mp 255-257 ${ }^{\circ} \mathrm{C}$, FT-IR $\left(\mathrm{KBr}, \mathrm{cm}^{-1}\right): 1645$ ( $\mathrm{C}=\mathrm{O}$ stretching), 1598 (aromatic $\mathrm{C}=\mathrm{C}$ or $\mathrm{N}=\mathrm{N}$ streching), 1450 (aromatic $\mathrm{C}=\mathrm{C}$ stretching), $1375 \quad(\mathrm{C}-\mathrm{N}$ or $\mathrm{C}-\mathrm{S}$ stretching) $\mathrm{cm}^{-1} \cdot{ }^{1} \mathrm{H}$ NMR (DMSO-d $\left.6,500 \mathrm{MHz}\right): \delta_{\mathrm{H}} ; 3.00(\mathrm{~s}, 6 \mathrm{H}$, $2 \mathrm{CH} 3-\mathrm{N}), 4.49(\mathrm{~s}, 2 \mathrm{H}), 6.10(\mathrm{~s}, 1 \mathrm{H}), 6.67(\mathrm{~d}, J=8.2 \mathrm{~Hz}, 2 \mathrm{H}), 6.78$ $(\mathrm{d}, J=8.5 \mathrm{~Hz}, 1 \mathrm{H}), 7.40(\mathrm{~d}, J=8.7 \mathrm{~Hz}, 2 \mathrm{H}), 7.50(\mathrm{t}, J=7.9 \mathrm{~Hz}$, $3 \mathrm{H}), 7.67$ (t, $J=10.2 \mathrm{~Hz}, 3 \mathrm{H}), 7.74(\mathrm{dd}, J=7.2 \mathrm{~Hz}, J=1.3 \mathrm{~Hz}$, $2 \mathrm{H}) \mathrm{ppm}$. HR-MS $\left(\mathrm{m} / \mathrm{z}\right.$ 402). Anal. calcd for $\mathrm{C}_{23} \mathrm{H}_{22} \mathrm{~N}_{4} \mathrm{OS}$ : C, 68.63; H, 5.51; N, 13.92. Found: C, 68.66; H, 5.54; N, 13.94.

2-(4-Methoxyphenyl)-3-(4-(phenyldiazenyl)phenyl)

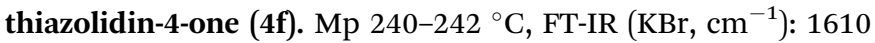
( $\mathrm{C}=\mathrm{O}$ stretching), 1586 (aromatic $\mathrm{C}=\mathrm{C}$ or $\mathrm{N}=\mathrm{N}$ stretching), 1500, 1404 (C-S or C-O stretching), 1300 (C-N stretching) $\mathrm{cm}^{-1}$. ${ }^{1} \mathrm{H}$ NMR (DMSO-d $\left.{ }_{6}, 500 \mathrm{MHz}\right): \delta_{\mathrm{H}} ; 3.73\left(\mathrm{~s}, 3 \mathrm{H}, \mathrm{OCH}_{3}\right), 4.40(\mathrm{~s}$, $2 \mathrm{H}), 6.10(\mathrm{~s}, 1 \mathrm{H}), 6.85(\mathrm{~d}, J=8.4 \mathrm{~Hz}, 2 \mathrm{H}), 7.00(\mathrm{~d}, J=6.0 \mathrm{~Hz}, 1 \mathrm{H})$, $7.09-7.13(\mathrm{~m}, 2 \mathrm{H}), 7.29$ (d, $J=8.4 \mathrm{~Hz}, 2 \mathrm{H}), 7.42(\mathrm{~d}, J=8.3 \mathrm{~Hz}$, $1 \mathrm{H}), 7.54-7.67(\mathrm{~m}, 2 \mathrm{H}), 7.74(\mathrm{~d}, J=8.1 \mathrm{~Hz}, 1 \mathrm{H}), 7.88(\mathrm{t}, J=$ $9.4 \mathrm{~Hz}, 1 \mathrm{H}), 7.94(\mathrm{t}, J=9.6 \mathrm{~Hz}, 1 \mathrm{H}) \mathrm{ppm} .{ }^{13} \mathrm{C}$ NMR (DMSO-d6, $125 \mathrm{MHz}): \delta_{\mathrm{C}} ; 50.89,55.40,55.51,122.48,123.03,123.59$, 123.89, 127.93, 129.48, 129.67, 130.23, 130.34, 130.89, 131.85,

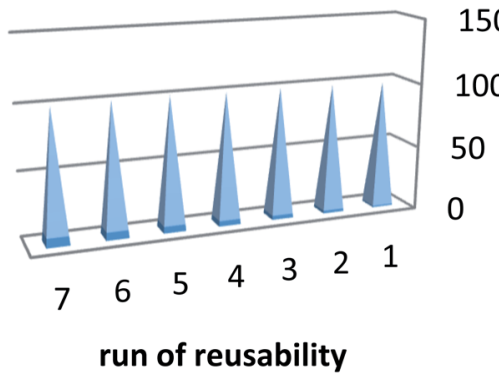

150

100

Yield (\%)

Fig. 1 The reusability of catalyst. 
147.89, 191.37 ppm. HR-MS $(\mathrm{m} / z$ 389). Anal. calcd for $\mathrm{C}_{22} \mathrm{H}_{19} \mathrm{~N}_{3} \mathrm{O}_{2} \mathrm{~S}: \mathrm{C}, 67.84 ; \mathrm{H}, 4.92 ; \mathrm{N}, 10.79$. Found: C, 67.86; H, $4.94 ; \mathrm{N}, 10.75$.

2-(3-Chlorophenyl)-3-(4-(phenyldiazenyl)phenyl)thiazolidin4-one (4g). $\mathrm{Mp} 231-233{ }^{\circ} \mathrm{C}$, FT-IR $\left(\mathrm{KBr}, \mathrm{cm}^{-1}\right): \delta_{\mathrm{H}} ; 1631(\mathrm{C}=\mathrm{O}$ stretching), 1594 (aromatic $\mathrm{C}=\mathrm{C}$ stretching or $\mathrm{N}=\mathrm{N}$ streching), 1450 (aromatic $\mathrm{C}=\mathrm{C}$ stretching), 1330 (C-N or C-S stretching), 1150 (C-Cl stretching). ${ }^{1} \mathrm{H}$ NMR (DMSO-d $\left.{ }_{6}, 500 \mathrm{MHz}\right): \delta_{\mathrm{H}} ; 4.51$ $(\mathrm{s}, 2 \mathrm{H}), 6.13(\mathrm{~s}, 1 \mathrm{H}), 6.89(\mathrm{dd}, J=3.5,14.5 \mathrm{~Hz}, 3 \mathrm{H}), 7.31-7.45(\mathrm{~m}$, $2 \mathrm{H}), 7.52(\mathrm{t}, J=11.5 \mathrm{~Hz}, 2 \mathrm{H}), 7.68(\mathrm{dd}, J=3.0,17.5 \mathrm{~Hz}, 2 \mathrm{H}), 7.75$ (d, $J=8.5 \mathrm{~Hz}, 2 \mathrm{H}), 7.79-7.90(\mathrm{~m}, 2 \mathrm{H}) \mathrm{ppm} .{ }^{13} \mathrm{C}$ NMR (DMSO-d ${ }_{6}$, $75 \mathrm{MHz}): \delta_{\mathrm{C}} ; 57.21,59.63,113.90,115.63,122.17,122.88,125.62$, 128.01, 129.29, 129.61, 129.67, 129.89, 132.55, 143.26, 152.83, 153.32, 171.03 ppm. HR-MS (m/z 393). Anal. calcd for $\mathrm{C}_{21} \mathrm{H}_{16}{ }^{-}$ $\mathrm{ClN}_{3} \mathrm{OS}$ : C, 64.03; H, 4.09; N, 10.67. Found: C, 64.06; H, 4.07; N, 10.65 .

2-(3-Nitrophenyl)-3-(4-(phenyldiazenyl)phenyl)thiazolidin-4one (4h). Mp 205-207 ${ }^{\circ} \mathrm{C}$, FT-IR $\left(\mathrm{KBr}, \mathrm{cm}^{-1}\right): 1641(\mathrm{C}=\mathrm{O}$ stretching), 1600 (aromatic $\mathrm{C}=\mathrm{C}$ stretching), $1580 \quad(\mathrm{~N}=\mathrm{N}$ stretching or $\mathrm{NO}_{2}$ asymmetric stretching), $1483(\mathrm{C}=\mathrm{C}$ aromatic stretching), 1336 (C-N or C-S stretching or $\mathrm{NO}_{2}$ symmetric stretching), $1150 \mathrm{~cm}^{-1} .{ }^{1} \mathrm{H}$ NMR (DMSO-d 6 , $\left.500 \mathrm{MHz}\right): \delta_{\mathrm{H}} ; 4.65$ $(\mathrm{s}, 2 \mathrm{H}), 6.14(\mathrm{~s}, 1 \mathrm{H}), 6.70(\mathrm{~d}, J=8.0 \mathrm{~Hz}, 3 \mathrm{H}), 7.33-7.45(\mathrm{~m}, 1 \mathrm{H})$, $7.52(\mathrm{t}, J=11.0 \mathrm{~Hz}, 2 \mathrm{H}), 7.58(\mathrm{t}, J=13.0 \mathrm{~Hz}, 1 \mathrm{H}), 7.68(\mathrm{~d}, J=$ $9.0 \mathrm{~Hz}, 2 \mathrm{H}), 7.76(\mathrm{~d}, J=13.5 \mathrm{~Hz}, 3 \mathrm{H}), 7.87-7.92(\mathrm{~m}, 1 \mathrm{H}) \mathrm{ppm} .{ }^{13} \mathrm{C}$ NMR (DMSO-d 6 , $75 \mathrm{MHz}): \delta_{\mathrm{C}} ; 55.83,62.10,113.92,121.98$, 122.17 (two peaks), 123.08, 125.63, 129.65, 133.43, 136.76, 143.29, 145.63, 148.24, 148.83, 153.33, 176.53 ppm. HR-MS $(\mathrm{m} / z$ 393). Anal. calcd for $\mathrm{C}_{21} \mathrm{H}_{16} \mathrm{~N}_{4} \mathrm{O}_{3} \mathrm{~S}$ : C, 62.36; H, 3.99; N, 13.85 . Found: C, 62.39; H, 3.97; N, 13.86.

2-(2-Chlorophenyl)-3-(4-(phenyldiazenyl)phenyl)thiazolidin-

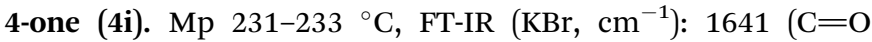
stretching), 1581 (aromatic $\mathrm{C}=\mathrm{C}$ stretching or $\mathrm{N}=\mathrm{N}$ stretching), 1446 (aromatic $\mathrm{C}=\mathrm{C}$ stretching), 1235 (C-N or $\mathrm{C}-\mathrm{S}$ stretching), 1173 (C-Cl stretching) $\mathrm{cm}^{-1} \cdot{ }^{1} \mathrm{H}$ NMR (DMSO-d ${ }_{6}$, $300 \mathrm{MHz}): \delta_{\mathrm{H}} ; 4.58(\mathrm{~s}, 2 \mathrm{H}), 6.12(\mathrm{~s}, 1 \mathrm{H}), 6.70(\mathrm{~d}, J=8.7 \mathrm{~Hz}, 1 \mathrm{H})$, 7.24-7.28 (m, 1H), 7.29-7.343 (m, 1H), 7.347-7.394 (m, 1H), 7.39-7.430 (m, 1H), 7.438-7.450 (m, 1H), 7.455-7.503 (m, 1H), 7.508-7.556 (m, 2H), 7.559-7.60 (m, 2H), 7.61-7.67 (m, 1H), 7.71 $(\mathrm{d}, J=14.4 \mathrm{~Hz}, 1 \mathrm{H}) \mathrm{ppm} .{ }^{13} \mathrm{C}$ NMR $\left(\mathrm{DMSO}_{6}, 75 \mathrm{MHz}\right): \delta_{\mathrm{C}}$; 60.69 (two peaks), 113.89, 122.18, 122.67, 122.91, 125.64, 127.48, 128.70, 129.06, 129.17, 129.90, 131.40, 140.03, 142.48, 153.69, 173.46 ppm. HR-MS $(m / z$ 404). Anal. calcd for $\mathrm{C}_{21} \mathrm{H}_{16} \mathrm{~N}_{4} \mathrm{O}_{3} \mathrm{~S}$ : C, 62.36; H, 3.99; N, 13.85. Found: C, 62.38; H, $3.97 ; \mathrm{N}, 13.88$.

2-(3-Bromophenyl)-3-(4-(phenyldiazenyl)phenyl)thiazolidin4-one (4j). Mp 139-141 ${ }^{\circ} \mathrm{C}$, FT-IR $\left(\mathrm{KBr}, \mathrm{cm}^{-1}\right): 1625(\mathrm{C}=\mathrm{O}$ stretching), 1590 (aromatic $\mathrm{C}=\mathrm{C}$ or $\mathrm{N}=\mathrm{N}$ stretching), 1383 (C$\mathrm{N}$ or $\mathrm{C}-\mathrm{S}$ or $\mathrm{C}-\mathrm{Br}$ stretching) $\mathrm{cm}^{-1} \cdot{ }^{1} \mathrm{H}$ NMR (DMSO-d 6,500 $\mathrm{MHz}): \delta_{\mathrm{H}} ; 4.49(\mathrm{~s}, 2 \mathrm{H}), 6.10(\mathrm{~s}, 1 \mathrm{H}), 6.67(\mathrm{~d}, J=8.8 \mathrm{~Hz}, 1 \mathrm{H}), 7.26-$ $7.31(\mathrm{~m}, 2 \mathrm{H}), 7.39-7.42(\mathrm{~m}, 1 \mathrm{H}), 7.50$ (t, $J=7.4 \mathrm{~Hz}, 3 \mathrm{H}), 7.59-$ $7.66(\mathrm{~m}, 1 \mathrm{H}), 7.73(\mathrm{~d}, J=8.5 \mathrm{~Hz}, 1 \mathrm{H}), 7.76(\mathrm{~d}, J=5.1 \mathrm{~Hz}, 2 \mathrm{H})$, $7.91(\mathrm{~d}, J=8.2 \mathrm{~Hz}, 1 \mathrm{H}), 7.98$ (d, $J=6.8 \mathrm{~Hz}, 1 \mathrm{H}) \mathrm{ppm}$. HR-MS $(\mathrm{m} / \mathrm{z}$ 439). Anal. calcd for $\mathrm{C}_{21} \mathrm{H}_{16} \mathrm{~N}_{3} \mathrm{SOBr}$ : C, 57.54; H, 3.68; N, 9.59. Found: C, 57.57; H, 3.67; N, 9.57.

2-(2-Nitrophenyl)-3-(4-(phenyldiazenyl)phenyl)thiazolidin-4one (4k). Mp 156-158 ${ }^{\circ} \mathrm{C}$, FT-IR $\left(\mathrm{KBr}, \mathrm{cm}^{-1}\right): 1632(\mathrm{C}=\mathrm{O}$ stretching), 1600 (aromatic $\mathrm{C}=\mathrm{C}$ or $\mathrm{N}=\mathrm{N}$ stretching), 1550 ( $\mathrm{NO}_{2}$ asymmetric stretching), 1518 (aromatic $\mathrm{C}=\mathrm{C}$ stretching), 1391 (NO2 symmetric stretching) $\mathrm{cm}^{-1} \cdot{ }^{1} \mathrm{H}$ NMR (DMSO-d 6 , 500 $\mathrm{MHz}): \delta_{\mathrm{H}} ; 3.53(\mathrm{~s}, 2 \mathrm{H}), 6.10(\mathrm{~s}, 1 \mathrm{H}), 6.67(\mathrm{~s}, 2 \mathrm{H}), 7.14(\mathrm{t}, J=$ $8.5 \mathrm{~Hz}, 1 \mathrm{H}), 7.35(\mathrm{t}, J=8.4 \mathrm{~Hz}, 1 \mathrm{H}), 7.40(\mathrm{t}, J=7.3 \mathrm{~Hz}, 1 \mathrm{H}), 7.50$ $(\mathrm{t}, J=8.0 \mathrm{~Hz}, 2 \mathrm{H}), 7.57-7.59(\mathrm{~m}, 1 \mathrm{H}), 7.65(\mathrm{~d}, J=8.7 \mathrm{~Hz}, 2 \mathrm{H})$, $7.73(\mathrm{~d}, J=9.3 \mathrm{~Hz}, 2 \mathrm{H}), 7.88$ (d, $J=7.5 \mathrm{~Hz}, 1 \mathrm{H}) \mathrm{ppm} .{ }^{13} \mathrm{C} \mathrm{NMR}$ $\left(\mathrm{DMSO}-\mathrm{d}_{6}, 125 \mathrm{MHz}\right): \delta_{\mathrm{C}} ; 54.59,55.10,113.45$ (two peaks), 121.75, 122.50, 123.99, 125.21, 129.24 (two peaks), 129.45, 141.09, 142.84, 147.16, 152.50, 152.93, 168.41 ppm. HR-MS $(\mathrm{m} / \mathrm{z}$ 393). Anal. calcd for $\mathrm{C}_{21} \mathrm{H}_{16} \mathrm{~N}_{4} \mathrm{O}_{3} \mathrm{~S}$ : C, 62.36; H, 3.99; N, 13.85 . Found: C, 62.35; H, 3.95; N, 13.89.

2-(2,4-Dichlorophenyl)-3-(4-(phenyldiazenyl)phenyl) thiazolidin-4-one (41). $\mathrm{Mp} 246-248{ }^{\circ} \mathrm{C}$, FT-IR $\left(\mathrm{KBr}, \mathrm{cm}^{-1}\right): 1645$ $(\mathrm{C}=\mathrm{O}$ stretching), 1581 (aromatic $\mathrm{C}=\mathrm{C}$ stretching or $\mathrm{N}=\mathrm{N}$ stretching), 1446 (aromatic $\mathrm{C}=\mathrm{C}$ stretching), 1350 (C-N or C-S stretching), 1141, 1091 (C-Cl stretching). ${ }^{1} \mathrm{H}$ NMR (DMSO-d ${ }_{6}$, $500 \mathrm{MHz}): \delta_{\mathrm{H}} ; 4.54(\mathrm{~s}, 2 \mathrm{H}), 6.13(\mathrm{~s}, 1 \mathrm{H}), 7.39-7.46(\mathrm{~m}, 2 \mathrm{H}), 7.50-$ $7.55(\mathrm{~m}, 1 \mathrm{H}), 7.56-7.65(\mathrm{~m}, 2 \mathrm{H}), 7.66-7.73(\mathrm{~m}, 1 \mathrm{H}), 7.74-7.78$ $(\mathrm{m}, 2 \mathrm{H}), 7.83(\mathrm{~d}, J=6.5 \mathrm{~Hz}, 1 \mathrm{H}), 7.88-7.94(\mathrm{~m}, 2 \mathrm{H}), 7.99(\mathrm{~d}, J=$ $14.5 \mathrm{~Hz}, 1 \mathrm{H}) \mathrm{ppm} .{ }^{13} \mathrm{C}$ NMR (DMSO-d $6,75 \mathrm{MHz}$ ): $\delta_{\mathrm{C}} ; 60.24$, $61.33,122.75,122.92,123.01,123.49,124.36,125.91,127.66$, $128.60,128.67,129.36,129.85,132.27,139.23,152.48$, 179.64 ppm. HR-MS $(m / z 427)$. Anal. calcd for $\mathrm{C}_{21} \mathrm{H}_{15} \mathrm{Cl}_{2} \mathrm{~N}_{3} \mathrm{OS}: \mathrm{C}$, 58.89; H, 3.53; N, 9.81. Found: C, 58.85; H, 3.56; N, 9.82.

2-(4-Hydroxy-3-methoxyphenyl)-3-(4-(phenyldiazenyl)phenyl) thiazolidin-4-one (4m). $\mathrm{Mp}>300{ }^{\circ} \mathrm{C}$, FT-IR $\left(\mathrm{KBr}, \mathrm{cm}^{-1}\right): 3206$ (O-H stretching), 1632 ( $\mathrm{C}=\mathrm{O}$ stretching), 1600 (aromatic $\mathrm{C}=\mathrm{C}$ stretching or $\mathrm{N}=\mathrm{N}$ stretching), 1450 (aromatic $\mathrm{C}=\mathrm{C}$ stretching), 1400 (C-N or C-S stretching), 1235 (C-O stretching). ${ }^{1} \mathrm{H}$ NMR (DMSO-d 6 , $300 \mathrm{MHz}): \delta_{\mathrm{H}} ; 3.69\left(\mathrm{~s}, 3 \mathrm{H}, \mathrm{OCH}_{3}\right), 4.41(\mathrm{~s}, 2 \mathrm{H})$, $6.15(\mathrm{~s}, 2 \mathrm{H}), 7.42(\mathrm{~d}, J=5.1 \mathrm{~Hz}, 1 \mathrm{H}), 7.49-7.56(\mathrm{~m}, 2 \mathrm{H}), 7.60(\mathrm{~d}, J$ $=4.5 \mathrm{~Hz}, 1 \mathrm{H}), 7.68(\mathrm{~d}, J=2.7 \mathrm{~Hz}, 2 \mathrm{H}), 7.75(\mathrm{~d}, J=7.2 \mathrm{~Hz}, 2 \mathrm{H})$, 7.88-7.95 (m, 2H) ppm. ${ }^{13} \mathrm{C} \mathrm{NMR}\left(\mathrm{DMSO}_{6}, 75 \mathrm{MHz}\right): \delta_{\mathrm{C}} ; 55.12$, 60.13, $67.82\left(\mathrm{OCH}_{3}\right), 113.91,117.43,119.94,122.17,122.92$, 123.03, 125.08, 129.67, 129.88, 143.25, 151.63, 152.42, 152.89, 153.36, 176.53 ppm. HR-MS $(\mathrm{m} / \mathrm{z}$ 405). Anal. calcd for $\mathrm{C}_{22} \mathrm{H}_{19} \mathrm{~N}_{3} \mathrm{O}_{3} \mathrm{~S}$ : C, 65.17; H, 4.72; N, 10.36. Found: C, 65.18; $\mathrm{H}$, $4.76 ; \mathrm{N}, 10.34$.

2-(3-(4-Chlorophenyl)-1-phenyl-1H-pyrazol-4-yl)-3-(4methoxyphenyl) thiazolidin-4-one (4n). Oil, FT-IR $\left(\mathrm{KBr}, \mathrm{cm}^{-1}\right)$ : 3126 (C-H aromatic), 2981 (C-H aliphatic), 1731 (C=O stretch), $1600,1541,1502,1342,1156 .{ }^{1} \mathrm{H}$ NMR (DMSO-d 6 , $\left.500 \mathrm{MHz}\right): \delta_{\mathrm{H}}$; $3.28(\mathrm{~d}, J=15.3 \mathrm{~Hz}, 1 \mathrm{H}), 3.5(\mathrm{~d}, J=15.3 \mathrm{~Hz}, 1 \mathrm{H}), 3.52(\mathrm{~s}, 3 \mathrm{H}$, $\left.\mathrm{CH}_{3} \mathrm{O}\right), 5.48(\mathrm{~s}, 1 \mathrm{H}), 7.32(\mathrm{t}, J=7.4 \mathrm{~Hz}, 1 \mathrm{H}), 7.43-7.49(\mathrm{~m}, 4 \mathrm{H})$, 7.73-7.80 (m, 4H), $8.22(\mathrm{~s}, 1 \mathrm{H}) \mathrm{ppm} .{ }^{13} \mathrm{C}$ NMR (DMSO-d 6 , 75 $\mathrm{MHz}): \delta_{\mathrm{C}} ; 32.9,40.4,60.5,118.0,125.5,127.4,127.8,128.4$, 128.7, 129.5, 129.8, 133.6, 138.8, 143.1, 168.3 (C=O) ppm.

3-(4-Methoxyphenyl)-2-(1,3-diphenyl-1H-pyrazol-4-yl) thiazolidin-4-one (4o). Oil, FT-IR $\left(\mathrm{KBr}, \mathrm{cm}^{-1}\right)$ : $3058(\mathrm{C}-\mathrm{H}$ aromatic), 2977 (C-H aliphatic), 1737 (C=O stretch), $1596(\mathrm{C}=$ C, aromatic), 1541, 1500, 1288 (C-O). ${ }^{1} \mathrm{H}$ NMR (DMSO-d 6,500 MHz): $\delta_{\mathrm{H}} ; 3.29(\mathrm{~d}, J=15.2 \mathrm{~Hz}, 1 \mathrm{H}), 3.49(\mathrm{~d}, J=15.2 \mathrm{~Hz}, 1 \mathrm{H}), 3.58$ (s, 3H, CH3O), 5.49 (s, 1H), $7.30(\mathrm{td}, J=7.9 \mathrm{~Hz}, 0.7 \mathrm{~Hz}, 2 \mathrm{H})$, 7.37-7.41 (m, 2H), 7.45-7.48 (m, 6H), $7.76(\mathrm{dd}, J=8.4,0.6 \mathrm{~Hz}$, $2 \mathrm{H}), 7.8(\mathrm{~d}, J=8.4 \mathrm{~Hz}, 2 \mathrm{H}), 8.09(\mathrm{~s}, 1 \mathrm{H}) .{ }^{13} \mathrm{C}$ NMR $\left(D_{M S O}-\mathrm{d}_{6}, 75\right.$ 
$\mathrm{MHz}): \delta_{\mathrm{C}} ; 32.9,43.5,60.5,118.0,125.7,127.1,127.3,127.4$, 127.5, 127.6, 128.4, 131.3, 138.6, 150.0, $168.8(\mathrm{C}=\mathrm{O}) \mathrm{ppm}$.

3-(2-Methyl-4-nitrophenyl)-2-(1,3-diphenyl-1H-pyrazol-4-yl) thiazolidin-4-one (4p). Oil, FT-IR $\left(\mathrm{KBr}, \mathrm{cm}^{-1}\right): 3126(\mathrm{C}-\mathrm{H}$ aromatic), 2981 (C-H aliphatic), 1730 (C=O stretch), 1596 (C= $\mathrm{C}$ aromatic), $1541\left(\mathrm{NO}_{2}\right), 1500,1450,1363 .{ }^{1} \mathrm{H}$ NMR $\left(\mathrm{DMSO}_{6} \mathrm{~d}_{6}\right.$, $500 \mathrm{MHz}): \delta_{\mathrm{H}} ; 3.30(\mathrm{~d}, J=15.15 \mathrm{~Hz}, 1 \mathrm{H}), 3.50(\mathrm{~d}, J=15.15 \mathrm{~Hz}$, $1 \mathrm{H}), 3.58\left(\mathrm{~s}, 3 \mathrm{H}, \mathrm{CH}_{3}\right), 5.50(\mathrm{~s}, 1 \mathrm{H}), 7.31(\mathrm{t}, J=7.4 \mathrm{~Hz}, 1 \mathrm{H}), 7.40-$ $7.42(\mathrm{~m}, 1 \mathrm{H}), 7.45-7.49(\mathrm{~m}, 7 \mathrm{H}), 7.75-7.78$ (m, 2H), 7.80-7.82 $(\mathrm{m}, 2 \mathrm{H}), 8.23(\mathrm{~s}, 1 \mathrm{H}) .{ }^{13} \mathrm{C}$ NMR $\left(\mathrm{DMSO}_{6}, 75 \mathrm{MHz}\right): \delta_{\mathrm{C}} ; 30.9$, 40.4 , 43.5, 60.5, 60.6, 118.0, 125.7, 127.1, 127.3, 127.4, 127.5, 127.6, 128.4, 131.3, 138.6, 150.0, $168.8(\mathrm{C}=\mathrm{O}) \mathrm{ppm}$.

2-(3-(4-Chlorophenyl)-1-phenyl-1H-pyrazol-4-yl)-3-(2-methyl4-nitrophenyl)thiazolidin-4-one (4q). Oil, FT-IR $\left(\mathrm{KBr}, \mathrm{cm}^{-1}\right)$ : 3128 (C-H aromatic), 2981 (C-H aliphatic), 1731 ( $\mathrm{C}=\mathrm{O}$ stretch), $1598\left(\mathrm{C}=\mathrm{C}\right.$ aromatic), $1539\left(\mathrm{NO}_{2}\right), 1500,1454,1400 \mathrm{~cm}^{-1} \cdot{ }^{1} \mathrm{H}$ NMR (DMSO-d $6,300 \mathrm{MHz}): \delta_{\mathrm{H}} ; 3.24(\mathrm{~d}, J=15.2 \mathrm{~Hz} 1 \mathrm{H}), 3.49(\mathrm{~d}, J$ $=15.2 \mathrm{~Hz} 1 \mathrm{H}), 3.55\left(\mathrm{~s}, 1 \mathrm{H}, \mathrm{CH}_{3}\right), 5.45(\mathrm{~s}, 1 \mathrm{H}), 7.26-7.30(\mathrm{~m}$, $2 \mathrm{H}), 7.40-7.45(\mathrm{~m}, 6 \mathrm{H}), 7.71-7.73(\mathrm{~m}, 2 \mathrm{H}), 7.75-7.78(\mathrm{~m}, 2 \mathrm{H})$, $8.20(\mathrm{~s}, 1 \mathrm{H}) \mathrm{ppm} .{ }^{13} \mathrm{C}$ NMR (DMSO-d 6 , $\left.75 \mathrm{MHz}\right): \delta_{\mathrm{C}} ; 32.7,40.3$, 43.2, 60.4, 60.5, 117.8, 117.9, 125.7, 127.3, 127.6, 128.3, 128.6, 129.7, 133.1, 138.4, 148.7, $168.1(\mathrm{C}=\mathrm{O}) \mathrm{ppm}$.

\section{Conflicts of interest}

There are no conflicts to declare.

\section{Acknowledgements}

Financial support from the Research Council of Payame Noor University of Rasht and Kerman branch is sincerely acknowledged.

\section{References}

1 F. W. A. Barros, T. G. Silva, M. G. da Rocha Pitta, D. P. Bezerra, L. V. Costa-Lotufo, M. O. de Moraes, C. Pessoa, M. A. F. B. de Moura, F. C. de Abreu, M. C. A. de Lima, S. linsGaldino, I. da Rocha Pitta and M. O. F. Goulart, Bioorg. Med. Chem., 2014, 20, 3533.

2 F. E. Onen-Bayram, I. Durmaz, D. Scherman, J. Herscovici and R. Cetin-Atalay, Bioorg. Med. Chem., 2012, 20, 5094.

3 M. S. A. El-Gaby, Z. H. Ismail, S. M. Abdel-Gawad, H. M. Aly and M. M. Ghorab, Phosphorus, Sulfur, Silicon Relat. Elem., 2009, 184, 2645.

4 Z. Beharry, M. S. Mahajan, F. Zhang, J. Ma, Z. Xia, M. Lilly, C. D. Smith and A. S. Kraft, Mol. Cancer Ther., 2009, 8, 1473.

5 N. C. Desai, K. M. Rajpara and V. V. Joshi, J. Fluorine Chem., 2013, 145, 102.

6 C. D. Barros, A. A. Amato, T. B. de Oliveira, K. B. R. Iannini, A. L. da Silva, T. G. da Silva, E. S. Leite, M. Z. Hernandes, M. C. A. de Lima, S. L. Galdino, F. deAssis Rocha Neves and I. da Rocha Pitta, Bioorg. Med. Chem., 2010, 18, 3805.

7 X. Xua, X. Qianb and L. Zhong, J. Fluorine Chem., 2005, 126, 297.
8 K. M. Orrling, M. R. Marzahn, H. Gutierrez-de-Teran, J. Aqvist, B. M. Dunn and M. Larhed, Bioorg. Med. Chem., 2009, 17, 5933.

9 G. Li, X. Qian, J. Cui, Q. Huang, D. Cui, R. Zhang and F. Liu, J. Fluorine Chem., 2006, 127, 182.

10 E. Ami, K. Nakahara, A. Sato, J.-T. Nguyen, K. Hidaka, Y. Hamada, S. Nakatani, T. Kimura, Y. Hayashia and Y. Kisoa, Bioorg. Med. Chem. Lett., 2007, 17, 4213.

11 W. Li, Y. Lu, Z. Wang, T. James, D. Dalton. and D. Miller, Bioorg. Med. Chem. Lett., 2007, 17, 4113.

12 M. D'Ascenzio, B. Bizzarri, C. De Monte, S. Carradori, A. Bolasco, D. Secci, D. Rivanera, N. Faulhaber, C. Bordón and L. Jones-Brando, Eur. J. Med. Chem., 2014, 86, 17.

13 R. K. Rawal, R. Tripathi, S. B. Katti, C. Pannecouque and E. D. Clercq, Bioorg. Med. Chem., 2007, 15, 1725.

14 M. R. Shiradkar, M. Ghodake, K. G. Bothara, S. V. Bhandari, A. Nikalje, K. ChakravarthyAkula, N. C. Desai and P. J. Burange, ARKIVOC, 2007, xiv, 58.

15 R. Ottana, E. Mazzon, L. Dugo, F. Monforte, R. Maccari, L. Sautebin, G. De Luca, M. G. Vigorita, S. Alcaro, F. Ortuso, A. P. Caputi and S. Cuzzocrea, Eur. J. Pharmacol., 2002, 448, 71.

16 M. V. Diurno, O. Mazzoni, G. Correale, I. G. Monterrey, A. Calignano, G. L. Rana and A. Bolognese, Il Farmaco, 1999, 54, 579.

17 Y. Tanabe, H. Yamamoto, M. Murakami, K. Yanagi, Y. Kubota, H. Okumura, Y. Sanemitsu and G. Suzukamo, J. Chem. Soc., Perkin Trans., 1995, 7, 935.

18 T. Kato, T. Ozaki, K. Tamura, Y. Suzuki, M. Akima and N. Ohi, J. Med. Chem., 1999, 42, 3134.

19 G. Marc, A. Stana, S. D. Oniga, A. Pîrnau, L. Vlase and O. Oniga, Molecules, 2019, 24, 2060.

20 T. L. da Silva, L. M. F. Miolo, F. S. S. Sousa, L. M. P. Brod, L. Savegnago and P. H. Schneider, Tetrahedron Lett., 2015, 56, 6674 .

21 B. P. Navin, R. P. Hemant, M. S. Faiyazalam and R. Dhanji, Med. Chem. Res., 2014, 23, 1360.

22 A. Deep, S. Jain, P. C. Sharma, P. Phogat and M. Malhotra, Med. Chem. Res., 2012, 21, 1652.

23 R. Markovic and M. Stodanovic, Heterocycles, 2005, 56, 2635. 24 R. B. Pawar and V. V. Mulwad, Chem. Heterocycl. Compd., 2004, 40, 219.

25 N. Ocal, F. Aydogan, C. Yolacan and Z. Turgut, J. Heterocycl. Chem., 2003, 40, 721.

26 O. S. Eltsov, V. S. Mokrushin, N. P. Belskaya and N. M. Kozlova, Russ. Chem. Bull., 2003, 52, 461.

27 M. H. Bolli, S. Abele, C. Binkert, R. Bravo, R. S. Buchmann, D. Bur, J. Gatfield, P. Hess, C. Kohl., C. Mangold, B. Mathys, M. Menyhart, C. Meuller, O. Nayler, M. Scherz, G. Schmidt, V. Sippel, B. Steiner, D. Strasser, A. Treiber and T. Weller, J. Med. Chem., 2010, 53, 4198.

28 P. T. Anastas and T. C. Williamson, Green Chemistry: Frontiers in Benign Chemical Syntheses and Processes, Oxford University Press, 1998.

29 M. Devprakash and U. A. Bhoi, J. Pharm. Res., 2011, 4, 2436. 30 M. Cacic, M. Molnar, B. Sarkanj, E. Has-Schon and V. Rajkovic, Molecules, 2010, 15, 6795. 
31 D. J. Adams, P. J. Dyson and S. J. Tavener, Chemistry in Alternative Reaction Media, John Wiley \& Sons, Chichester, UK, 2004.

32 P. Pollet, E. A. Davey, E. E. Ureña-Benavides, C. A. Eckert and C. L. Liotta, Green Chem., 2014, 16, 1034.

33 P. Wassercheid and T. Welton Ionic Liquids in Synthesis, Wiley-VCH, 2nd edn, New York, NY, USA, 2008.

34 L. Zhen, Z. Yingwei, H. Feng, Y. Lei, S. He Yuan, Ch. Jing and X. Change, Sci. Sin.: Chim., 2012, 42, 502.

35 T. L. Greaves and C. J. Drummond, Chem. Rev., 2008, 108, 206.

36 L. He, G. H. Tao, W. S. Liu, W. Xiong, T. Wang and Y. Kou, Chem. Lett., 2006, 17, 321.

37 A. Bado-Nilles, D. Alpha-Oumar, G. Marlair, P. Pandard, L. Chabot, A. Geffard, Ch. Len, J. M. Porcher and W. Sanchez, J. Hazard. Mater., 2015, 283, 202-210.

38 K. Ueno, R. Tatara, S. Tsuzuki, S. Saito, H. Doi, K. Yushida, T. Mandaei, M. Matsugami, Y. Umebayashi, K. Dokko and M. Watanabe, Phys. Chem. Chem. Phys., 2014, 16, 1967.

39 A. O. Diallo, G. Fayet, Ch. Len and G. Marlair, Ind. Eng. Chem. Res., 2012, 51, 3149.

40 A. O. Diallo, Ch. Len, A. B. Morgan and G. Marlair, Sep. Purif. Technol., 2012, 97, 228.

41 A. O. Diallo, A. B. Morgan, Ch. Len and G. Marlair, Energy Environ. Sci., 2013, 6, 699.
42 M. Nikpassand, L. Z. Fekri, M. Gharib and O. Marvi, Lett. Org. Chem., 2012, 9, 745.

43 L. Zare, N. Mahmoodi, A. Yahyazadeh, M. Mamaghani and K. Tabatabaeian, J. Heterocycl. Chem., 2011, 48, 864-867.

44 M. Nikpassand, L. Z. Fekri and S. Sanagou, Dyes Pigm., 2017, 136, 140.

45 L. Z. Fekri and R. Maleki,J. Heterocycl. Chem., 2017, 54, 1167. 46 L. ZareFekri, M. Nikpassand, Sh. Shariati, B. Aghazadeh and R. Zarkeshvari, J. Organomet. Chem., 2018, 871, 60.

47 L. ZareFekri, M. Nikpassand, S. Pourmirzajani and B. Aghazadeh, RSC Adv., 2018, 8, 22313.

48 L. ZareFekri, M. Nikpassand and S. NazariKhakshoor, J. Organomet. Chem., 2019, 894, 18.

49 L. Z. Fekri, M. Nikpassand and K. H. Pour, Curr. Org. Synth., 2015, 12, 76 .

50 L. Zare, N. O. Mahmoodi, A. Yahyazadeh, M. Mamaghani and K. Tabatabaeian, Chin. Chem. Lett., 2010, 21, 538.

51 L. Zare Fekri and M. Nikpassand, Russ. J. Gen. Chem., 2014, 84, 1825.

52 L. Z. Fekri and M. Nikpassand, Acta Chim. Slov., 2016, 63, 263.

53 M. Nikpassand, L. Z. Fekri and H. Taherkhorsand, Heterocycl. Commun., 2017, 23, 429. 